Journal for ImmunoTherapy of Cancer

\section{Safety of immune checkpoint inhibitor rechallenge after discontinuation for grade $\geq 2$ immune-related adverse events in patients with cancer}

To cite: Allouchery M, Lombard T, Martin M, et al. Safety of immune checkpoint inhibitor rechallenge after discontinuation for grade $\geq 2$ immune-related adverse events in patients with cancer. Journal for ImmunoTherapy of Cancer 2020;8:e001622. doi:10.1136/ jitc-2020-001622

- Additional material is published online only. To view please visit the journal online (http://dx.doi.org/10.1136/jitc2020-001622).

Accepted 27 November 2020

Check for updates

(c) Author(s) (or their employer(s)) 2020. Re-use permitted under CC BY-NC. No commercial re-use. See rights and permissions. Published by BMJ.

For numbered affiliations see end of article.

Correspondence to

Dr Mathieu Puyade;

mathieu.puyade@chu-poitiers.fr

\section{ABSTRACT}

Background Safety of rechallenge of immune checkpoint inhibitor (ICI) after grade $\geq 2$ immune-related adverse events (irAEs) leading to ICI discontinuation remains unclear.

Methods All adverse drug reactions involving at least one $\mathrm{ICl}$ reported up to December 31, 2019 were extracted from the French pharmacovigilance database. Patients were included if they experienced at least one grade $\geq 2$ irAE resulting in $\mathrm{ICl}$ discontinuation, with subsequent $\mathrm{ICl}$ rechallenge. The primary outcome was the recurrence of at least one grade $\geq 2$ irAE in these patients after ICl rechallenge.

Results We included 180 patients: $61.1 \%$ were men (median age of 66 years), $43.9 \%$ had melanoma and $78.9 \%$ were receiving anti-programmed cell death 1 . First ICI discontinuation was related to 191 irAEs. After $\mathrm{ICI}$ rechallenge, $38.9 \%$ of the patients experienced at least one grade $\geq 2$ irAE. Among them, $70.0 \%$ experienced the same irAE, $25.7 \%$ a distinct irAE, and $4.3 \%$ both the same and a distinct irAE. Lower recurrence rates of irAEs were associated with rechallenge with the same ICI treatment $(p=0.02)$ or first endocrine irAEs $(p=0.003)$. Gastrointestinal irAEs were more likely to recur $(p=0.007)$. The median duration from ICI discontinuation to rechallenge and the severity of the initial irAE did not predict recurrent irAEs after $\mathrm{ICI}$ rechallenge $(p=0.53$ and $p=0.40$, respectively).

Conclusions In this study, $61.1 \%$ of the patients who discontinued ICI treatment for grade $\geq 2$ irAEs experienced no recurrent grade $\geq 2$ irAEs after ICl rechallenge. Although $\mathrm{ICl}$ rechallenge appears to be safe under close monitoring, it should always be discussed balancing usefulness of rechallenge, patient comorbidities and risk of recurrence of first irAE(s). Due to inherent bias associated with pharmacovigilance studies, further prospective studies are needed to assess risk factors that may influence patient outcomes after ICI rechallenge.

\section{INTRODUCTION}

Immune checkpoint inhibitors (ICIs) are the new standard of care in the treatment of many different types of cancer. ${ }^{1}$ ICIs promote antitumor immune response by inhibiting cytotoxic T-lymphocyte antigen-4 (CTLA-4) or programmed cell death 1 or ligand 1 (PD-1/PDL-1) or both, leading to durable responses when compared with standard chemotherapy. $^{2-9}$ Because of the rapid and broad expansion of their indications, ICIs have been prescribed to a growing number of patients, resulting in increased understanding of their safety profile.

ICI use is associated with immune-related adverse events (irAEs), related to their T-cell mechanism of action. IrAEs can affect nearly any organ system, with varying degrees of severity. ${ }^{10}$ ICI therapy can generally be continued with close monitoring in the presence of grade 1 irAEs. Grade $\geq 2$ irAEs require corticosteroids and temporary or permanent ICI discontinuation. ${ }^{11-13}$ Severe irAEs leading to ICI discontinuation affect approximately $15 \%, 10 \%$ and $5 \%$ of patients receiving anti-CTLA4, anti-PD-1 or anti-PD-L1 agents, respectively, and $50 \%$ of patients receiving both nivolumab and ipilimumab. ${ }^{1011} 1314$ According to the current guidelines, permanent discontinuation of ICI treatment is generally recommended for grade 4 irAEs. The decision to resume ICI treatment after grade $\geq 2$ irAEs often remains challenging. Indeed, ICI rechallenge can be associated not only with significant antitumor response, ${ }^{15} 16$ but also with a theoretically increased significant risk of $\operatorname{irAE}(\mathrm{s})$ in comparison with naïve patients. Indeed, few studies have explored the issue of ICI rechallenge after an irAE. The largest series of ICI rechallenges after an irAE focused only on the recurrence rate of the same irAE after the rechallenge of the same ICI, thereby covering only part of the possible clinical scenario. ${ }^{17}$ Many other studies have also been based on small samples of patients. ${ }^{15161819}$ So far, the 
recurrence rate of irAEs varies from $39 \%$ to $55 \% .{ }^{20}$ In this study, we aimed to characterize the safety of ICI rechallenge after initial grade $\geq 2$ irAEs, which led to subsequent ICI discontinuation.

\section{METHODS}

\section{Data source}

Data were extracted from the French pharmacovigilance database (FPVD). Briefly, the FPVD includes all adverse drug reactions (ADRs) spontaneously reported to the 31 French Regional Pharmacovigilance Centers since 1985. French regulations require healthcare professionals to report all ADRs to the Regional Pharmacovigilance Centre on which they depend. All ADRs are diagnosed by healthcare specialists on the basis of clinical data, laboratory findings and/or imaging if necessary. All ADRs reports are then reviewed by pharmacologists with expertize in this field. Causality is assessed according to the French method for causality assessment of ADRs. ${ }^{21}$ Cases of ADRs are then recorded in the database, using the Medical Dictionary for Regulatory Activities. ${ }^{22}$

\section{Eligibility criteria}

All ADRs involving at least one ICI (including nivolumab, pembrolizumab, cemiplimab, ipilimumab, atezolizumab, durvalumab and avelumab) coded as suspected or interacting drugs according to the WHO criteria and reported up to December 31, 2019 were extracted from the FPVD. Patients were included if they experienced at least one grade $\geq 2$ irAE resulting in substantial ICI discontinuation, with subsequent ICI rechallenge. Patients must have discontinued ICI treatment for a period at least equal to twice the duration of a cycle (ie, for at least 4 weeks for a 2 weekly dosing regimen, 6 weeks for a 3 weekly dosing regimen, 8 weeks for a monthly dosing regimen and 12 weeks for a 6 weekly dosing regimen).

For each included case, the following data were collected: patient data (age, sex, body mass index (BMI)), characteristics of the ICIs (type, indication (including number of prior courses), duration between discontinuation and rechallenge, reason for ICI rechallenge), characteristics of the irAEs (type, time to occurrence, severity according to the National Cancer Institute Common Terminology Criteria for Adverse Events (NCI CTCAE) V.5.0, management (including corticosteroids and other immunosuppressive agents) and outcomes).

\section{Clinical outcomes}

The primary outcome was the recurrence of at least one grade $\geq 2$ irAE after ICI rechallenge in patients who discontinued ICI treatment for at least one grade $\geq 2$ irAE. We specifically evaluated the recurrence rate of the same grade $\geq 2$ irAE after ICI rechallenge, the recurrence rate of a distinct irAE after ICI rechallenge, and the characteristics of the patients and the irAEs. The recurrence of endocrine irAEs was defined as the need to increase significantly the dose of hormone replacement therapy

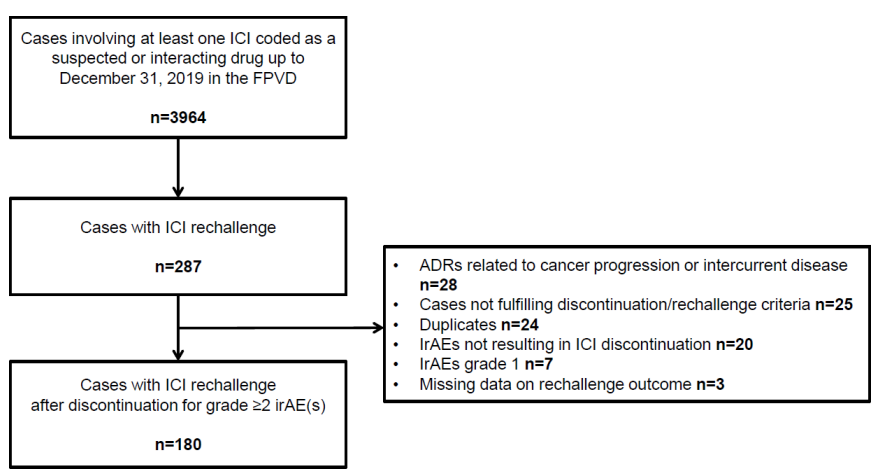

Figure 1 Flow chart. ADR, adverse drug reaction; FPVD, French pharmacovigilance database; ICl, immune checkpoint inhibitor; irAE, immune-related adverse event.

after ICI rechallenge. The factors associated with irAE recurrence after ICI rechallenge were considered as secondary outcomes.

\section{Statistical analysis}

In the descriptive statistical analyzes, continuous variables were described by mean and SD, or median and IQR and categorical variables by the number and proportion of subjects in each class. All variables with less than $20 \%$ of missing data were compared between groups using Student's t-test or Wilcoxon's test for continuous variables and Pearson's $\chi^{2}$ test or Fisher's exact test for categorical variables. The threshold for statistical significance was set at $\mathrm{p}<0.05$. All statistical analyzes were performed using SAS software (V.9.4).

\section{RESULTS \\ Description of the study population}

Patient and first ICI treatment characteristics

A total of 180 patients were included in the study (figure 1). Patient characteristics are summarized in table 1. Sixty-one per cent were men, the median (IQR) age was 66 (55-72) years. Melanoma was the most common tumor type $(43.9 \%)$, followed by lung cancer $(41.1 \%)$ and renal cell carcinoma $(6.1 \%)$. Seventy-nine per cent of the patients were receiving an anti-PD-1 agent, $10.0 \%$ an anti-CTLA- 4 and anti-PD- 1 combination, $6.0 \%$ an anti-CTLA-4 agent and 5.0\% an anti-PD-L1 agent. ICI treatment was initiated as a first-line treatment in $22.8 \%$ of the patients.

\section{Clinical features of grade $\geq 2$ irAEs leading to ICI discontinuation}

One hundred and ninety-one irAEs led to ICI discontinuation in 180 patients (table 2). Ten patients (5.6\%) had $\geq 2$ irAEs leading to ICI discontinuation. The most frequent irAEs resulting in ICI discontinuation included gastrointestinal disorders $(24.6 \%$, including colitis $(19.4 \%)$ and pancreatic disorders $(4.7 \%))$, endocrine disorders $(17.8 \%)$, hepatitis $(16.2 \%)$, respiratory disorders $(11.0 \%)$ and skin disorders $(9.4 \%)$. Fifty-two per cent of irAEs were grade $2,46.5 \%$ grade 3 and $1.5 \%$ grade 
Table 1 Characteristics of the study population

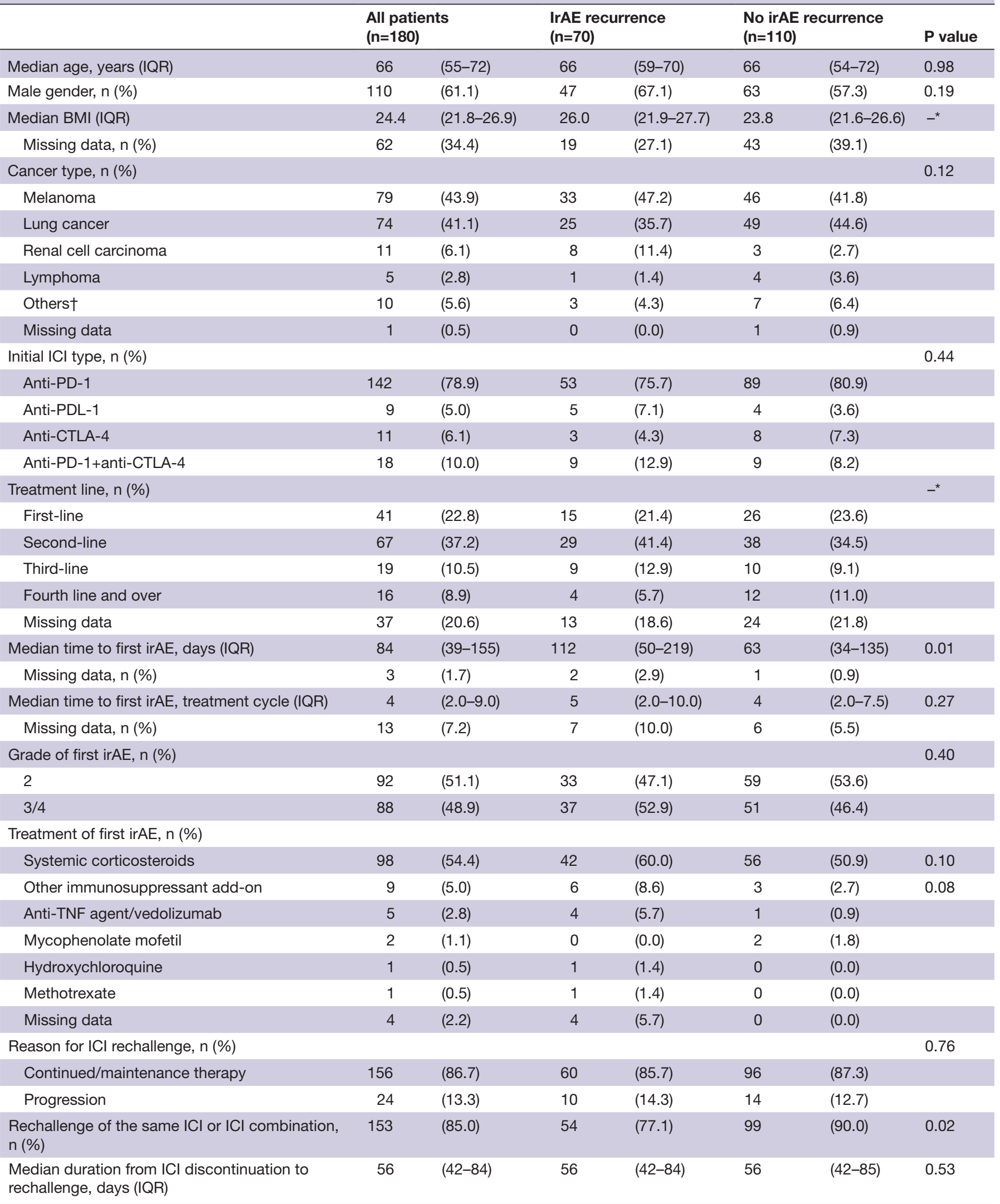

*More $>20.0 \%$ missing data.

tIncluding urothelial cancer $(n=3(1.7 \%))$, head and neck cancer $(n=2(1.1 \%))$, cutaneous squamous cell carcinoma $(n=2(1.1 \%))$, glioblastoma $(n=1$ $(0.5 \%))$, Merkel cell carcinoma $(n=1(0.5 \%))$ and colorectal cancer $(n=1(0.5 \%))$.

BMI, body mass index; CTLA-4, cytotoxic T-lymphocyte antigen-4; ICI, immune checkpoint inhibitor; irAE, immune related adverse event; PD-1, programmed cell death 1; PDL-1, programmed cell death 1 ligand 1; TNF, tumor necrosis factor. 
Table 2 Characteristics of the immune-related adverse events

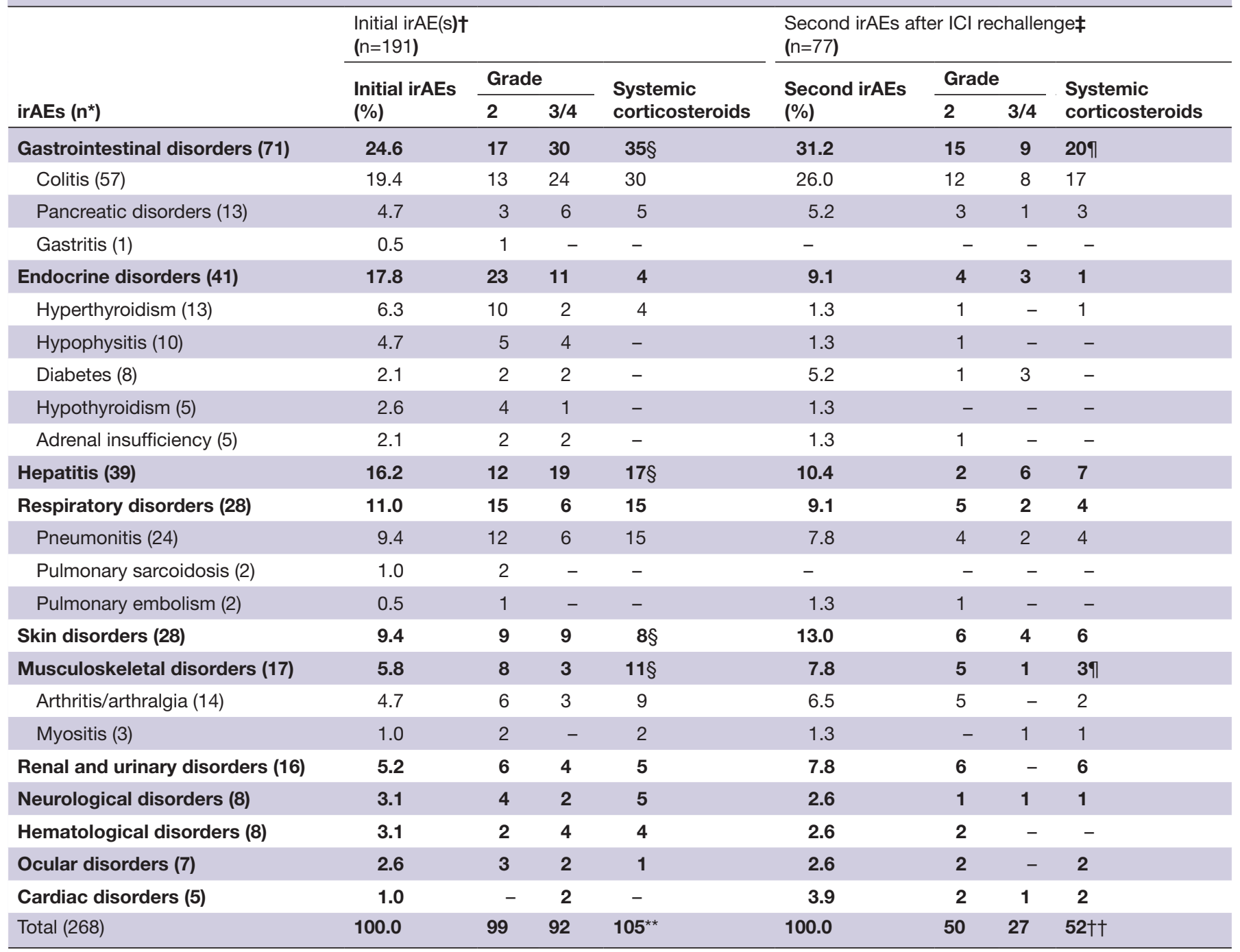

Detailed data on recurrent irAEs are shown in online supplemental table.

The bold values refer to organ involvement. Non bold values refer to more precise diseases.

*Including initial and second irAEs.

$\dagger 191$ grade $\geq 2$ irAEs led to discontinuation in 180 patients.

$\$ 77$ recurrent and/or new onset irAEs occurred in 70 patients.

§Immunosuppressive drugs were required in 11 initial irAEs (nine patients): colitis $(n=5)$, hepatitis $(n=3)$, arthritis $(n=2)$ and lichenoid dermatitis $(n=1)$.

IImmunosuppressive drugs were required in 6 second irAEs (six patients): colitis $(n=5)$ and arthritis $(n=1)$.

**Missing data in four initial irAEs.

††Missing data in 13 second irAEs.

$\mathrm{ICI}$, immune checkpoint inhibitor; IrAE, immune-related adverse event.

4. The median (IQR) time to occurrence of first irAE was 84 (39-155) days.

Among the 180 patients, $98(54.4 \%)$ received corticosteroid treatment. Among them, 9 (5.0\%) required immunosuppressive drugs, including anti-tumor necrosis factor (anti-TNF) agent or vedolizumab for colitis $(n=5$, $2.8 \%)$, mycophenolate mofetil for hepatitis $(\mathrm{n}=2,1.1 \%)$, hydroxychloroquine for lichenoid dermatitis $(\mathrm{n}=1,0.5 \%)$ and methotrexate for rheumatoid arthritis $(n=1,0.5 \%)$.

Characteristics of ICI rechallenge after discontinuation for grade $\geq 2$ irAE(s)

On resuming ICI after grade $\geq 2$ irAEs, 159 patients $(88.3 \%)$ received an anti-PD-1 agent and 153 patients
$(85.0 \%)$ the same ICI drug or the same ICIs combination. Ninety-one per cent of irAEs resolved to grade 1 or lower before ICI rechallenge. Fifty-two patients $(28.9 \%$, missing data $\mathrm{n}=23$ ) were on systemic corticosteroids when they resumed ICI treatment. One hundred and fifty-six patients $(86.7 \%)$ resumed ICI treatment as a maintenance therapy, and 24 (13.3\%) for disease progression. Median (IQR) duration from ICI discontinuation to rechallenge was 56 (42-84) days (table 1).

\section{Outcomes}

Safety of ICI rechallenge after discontinuation for grade $\geq 2$ irAE(s) After ICI rechallenge, 70 patients (38.9\%) experienced at least one grade $\geq 2 \operatorname{irAE}(n=77$ irAEs) (table 2). Six 


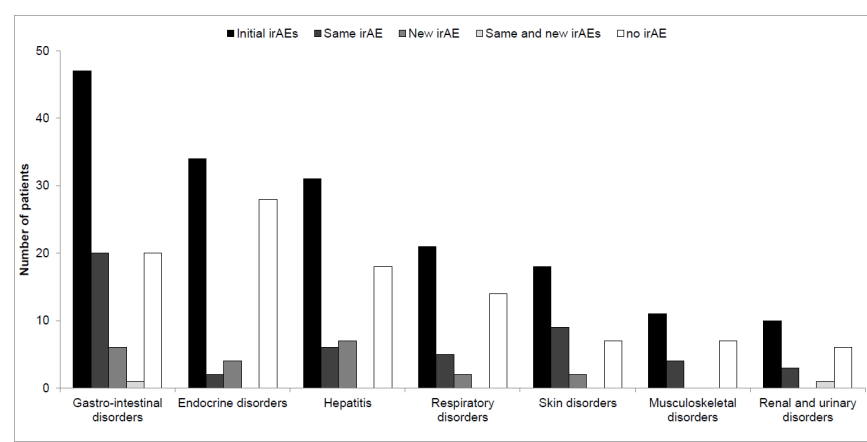

Figure 2 Patient outcomes after immune checkpoint inhibitor rechallenge according to the type of irAEs. irAE, immune-related adverse event.

patients $(8.6 \%)$ experienced $\geq 2$ recurrent (same or distinct) irAEs. Sixty-five per cent of irAEs were grade 2, $33.8 \%$ grade 3 and $1.2 \%$ grade 4 . The median (IQR) time to occurrence of the second irAE was 46 (16-103) days.

Among the 70 patients, $49(70.0 \%)$ experienced a recurrence of the first irAE, $18(25.7 \%)$ a new irAE, and 3 $(4.3 \%)$ both a recurrence of the first irAE and a new irAE after ICI rechallenge.

The recurrence rate of irAEs varied according to the organs involved in the first irAEs (online supplemental table). Twenty-one out of 47 patients (44.7\%) experienced recurrent gastrointestinal irAEs, 2 of $34(5.9 \%)$ recurrent endocrine irAEs, 9 of $18(50.0 \%)$ recurrent skin irAEs, 1 of $6(16.7 \%)$ recurrent encephalitis and 1 of $2(50.0 \%)$ recurrent myocarditis (figure 2 ).

Most patients $(68.6 \%)$ required systemic corticosteroids, and $6(8.6 \%)$ an immunosuppressive drug (anti-TNF agent or vedolizumab $(n=5)$ and methotrexate $(n=1))$. No death related to recurrent irAE was reported, and $76.6 \%$ of second irAEs resolved to grade 1 or lower. Forty-seven patients $(67.1 \%)$ discontinued ICI treatment due to recurrent irAEs.

\section{Factors associated with irAE(s) recurrence}

In patients with recurrent grade $\geq 2$ irAEs, the median time to first irAE was longer compared with patients without irAE after ICI rechallenge $(p=0.01)$ (table 1$)$. The patients rechallenged with the same ICI drug or ICIs combination had lower rates of recurrence than those rechallenged with another ICI drug or ICIs combination $(p=0.02)$. The patients with initial gastrointestinal irAEs were more likely to have recurrent grade $\geq 2$ irAEs after ICI rechallenge in comparison with other patients $(p=0.007)$. By contrast, initial endocrine irAEs were less likely to recur $(\mathrm{p}=0.003)$. The median duration from ICI discontinuation to rechallenge and the severity of the initial irAE did not predict recurrent irAEs after ICI rechallenge $(\mathrm{p}=0.53$ and $\mathrm{p}=0.40$, respectively).

\section{DISCUSSION}

This study aimed to provide complementary data on safety of ICI rechallenge after grade $\geq 2 \operatorname{irAE}(\mathrm{s})$, which have led to substantial ICI discontinuation in 180 patients. Herein, we found that $61.1 \%$ of the patients who discontinued ICI treatment for grade $\geq 2$ irAE $(\mathrm{s})$ experienced no recurrent grade $\geq 2$ irAE(s) after ICI rechallenge.

Data on safety of ICI rechallenge after grade $\geq 2$ irAE(s) leading to ICI discontinuation are scarce. Recent studies with small sample sizes were mainly focused on the rechallenge of anti-PD-1 or anti-PD-L1 after discontinuation for irAEs. Anti-PD-1 and anti-PDL-1 rechallenge was associated with occurrence of a second grade $\geq 2 \mathrm{irAE}$ in $55.0 \%$ of patients treated for various types of cancers. ${ }^{18}$ In 68 patients with anti-PD-L1 for advanced non-small cell lung cancer who stopped treatment due to irAEs, $55.0 \%$ experienced the same or a distinct irAE after antiPD-L1 rechallenge. ${ }^{15}$ In the largest published series of ICI rechallenges after irAEs $(\mathrm{n}=452)$, Dolladille et al explored recurrence of the same irAE after rechallenge with the same ICI and found a $28.8 \%$ recurrence rate of the same irAE, which is quite similar to the rate observed in our study. ${ }^{17}$

In the present study, no patient died after ICI rechallenge. Almost half of the patients experienced initial grade $\geq 3$ irAEs, while a third of the patients with recurrent irAEs experienced grade $\geq 3$ irAEs, suggesting that ICI rechallenge was not associated with more severe irAEs. Furthermore, we did not observe any statistically significant difference between the group with irAE recurrence and the group with no irAE recurrence with regard to first $\operatorname{irAE}(\mathrm{s})$ severity. ICI rechallenge should always be considered with caution after a grade 4 irAE. ${ }^{11}$ ICI rechallenge was associated with recurrence of a distinct irAE in 1 of 3 patients with initial grade 4 irAEs. In addition, due to life-threatening risk, ICI rechallenge after neurological or cardiac irAEs is not recommended. In our study, 1 out of 6 patients and 1 out of 2 patients experienced recurrent encephalitis and myocarditis respectively, raising the question of the benefit/risk ratio of ICI rechallenge in these patients.

In this study, the time to first irAE was longer in the recurrence group compared the free-recurrence group. By contrast, Simonaggio et al observed that the first irAEs occurred earlier in the patients who experienced new or recurrent irAEs after ICI rechallenge. ${ }^{18}$ Such discrepancy could be related to the wide profile of irAEs, and to the longer duration between ICI discontinuation and rechallenge in our cohort (median of 8 weeks vs 3.8 weeks). This latter aspect could lead to exclude early worsening of preexisting irAEs after ICI rechallenge, wrongly considered as irAEs recurrence.

Patients with initial gastrointestinal irAEs were more likely to have recurrent grade $\geq 2$ irAEs after ICI rechallenge compared with those with no gastrointestinal irAEs. By contrast, initial endocrine disorders were less likely to recur. These findings are consistent with those previously reported by Dolladille et al. ${ }^{17}$ These authors observed a higher recurrence rate for colitis compared with others irAEs, while the recurrence rate of adrenal irAEs was lower than that for other irAEs. 
The question of which ICI should be restarted after ICI discontinuation for severe irAEs is essential. In our study, the rechallenge of the same ICI drug or the same ICI combination was associated with a lower rate of irAE recurrence. This finding is particularly interesting for patients who progress after ICI discontinuation for irAE. Most studies have only reported the safety of PD-1 rechallenge as maintenance therapy after anti-PD-1/antiCTLA-4 discontinuation for severe irAEs. For example, Pollack et al concluded that patients who experienced colitis or hypophysitis after anti-PD-1/anti-CTLA-4 combination could safely resume anti-PD- $1 .{ }^{19}$ In a retrospective multicenter study in 167 patients, Abu-Sbeih et $a l$ also found that anti-PD-1/anti-PDL-1 rechallenge was associated with a lower risk of recurrent colitis (OR 0.30, $95 \%$ CI 0.11 to 0.81$).{ }^{23}$

This study has several strengths. First, all irAEs reports in the FPVD had been diagnosed by healthcare specialists and validated by a pharmacologist with expertize in the field. Second, this study provides additional data on safety of ICI rechallenge in a large cohort of patients treated in real-life practice with a broad spectrum of ICI regimens for varying types of cancers. It is also the first study to propose a standard definition of ICI rechallenge after discontinuation for irAEs. ICI rechallenge was only considered if the patients discontinued ICI treatment for a period at least equal to twice the duration of a cycle. Delyon et al suggested that the median duration between ICI discontinuation and rechallenge should be taken into account when considering ICI rechallenge. ${ }^{24}$ Indeed, as discussed earlier, a short temporary discontinuation could be associated with worsening of pre-existing irAEs, rather than recurrence of irAEs. However, in this study, the duration from ICI discontinuation to rechallenge was not associated with a higher risk of recurrence of irAEs.

Our study presents some limitations. The pharmacovigilance system is based on spontaneous reporting of ADRs, which can result in under reporting of less severe, for example, grade 2 irAEs, and/or expected ADRs. ${ }^{25} 26$ Given retrospective data collection, several data, such as number of prior courses or BMI were missing. Although the recurrence rate of irAEs in our study is quite similar to that previously reported, it could have been underestimated in the cases with insufficient retrospective update or when under-reporting concerned recurrent irAEs after ICI rechallenge. Despite these limitations, our results highlight the value of spontaneously reporting databases to characterize the safety profile of ICIs.

\section{CONCLUSIONS}

In conclusion, $61.1 \%$ of the patients who discontinued ICI treatment for grade $\geq 2$ irAEs experienced no recurrent grade $\geq 2$ irAEs after ICI rechallenge. ICI rechallenge after discontinuation for irAEs was not associated with more severe recurrent irAEs. Gastrointestinal disorders were more likely to recur after ICI rechallenge, whereas endocrine disorders had a lower recurrence rate. Due to inherent bias associated with pharmacovigilance studies, further prospective studies are needed to assess risk factors that influence patient outcomes after ICI rechallenge. Hence, even though ICI rechallenge appears to be safe under close monitoring, it should always be discussed in a multidisciplinary team meeting in light of usefulness of rechallenge, patient comorbidities and risk of recurrence of first $\operatorname{irAE}(\mathrm{s})$.

\section{Author affiliations}

${ }^{1}$ Pharmacologie clinique et Vigilances, Centre Hospitalier Universitaire de Poitiers, Poitiers, France

${ }^{2}$ Pharmacie à usage intérieur, Centre Hospitalier Universitaire de Poitiers, Poitiers, France

${ }^{3}$ Médecine Interne et Maladies Infectieuses, Centre Hospitalier Universitaire de Poitiers, Poitiers, France

${ }^{4}$ INSERM U1082, Centre Hospitalier Universitaire de Poitiers, Poitiers, France ${ }^{5}$ CRPV Marseille Provence Corse, service Hospitalo-Universitaire de Pharmacologie Clinique et Pharmacovigilance, Assistance Publique Hôpitaux de Marseille,

Marseille, France

${ }^{6}$ Centre Régional de PharmacoVigilance, CHU de Caen, Caen, France

${ }^{7}$ Département de Pharmacologie, CHU de Caen, Caen, France

${ }^{8}$ Département de Pharmacovigilance, Hôpital Henri Mondor, Creteil, France

${ }^{9}$ Service Hospitalo-Universitaire de Pharmaco-Toxicologie, Hospices Civils de Lyon, Lyon, France

${ }^{10}$ Univ. Bordeaux, INSERM, BPH, U1219, F-33000 Bordeaux, France

${ }^{11} \mathrm{CHU}$ de Bordeaux, Pole de Santé Publique, Pharmacologie Médicale, Centre de Pharmacovigilance de Bordeaux, Bordeaux, France

${ }^{12}$ Laboratoire de Neurosciences Expérimentales et Cliniques, INSERM U1084, Université de Poitiers, Poitiers, France

${ }^{13} \mathrm{CIC}-1402$, Centre Hospitalier Universitaire de Poitiers, Poitiers, France

Correction notice This paper has been updated since first published to amend affiliation 10 as 'Univ. Bordeaux, INSERM, BPH, U1219, F-33000 Bordeaux,France'.

Acknowledgements The authors would like to thank the 31 centers of the French pharmacovigilance network and Jeffrey Arsham for his help in the editing.

Contributors Conception and design: MA, TL, MP. Data collection: MA, TL. Analysis and interpretation of data: MA, TL, MP, M-CP-P. Writing, review and/or revision of the manuscript: all authors.

Funding The authors have not declared a specific grant for this research from any funding agency in the public, commercial or not-for-profit sectors.

Competing interests None declared.

Patient consent for publication Not required.

Ethics approval In accordance with French regulations, formal approval by an investigational review board is not required for this type of study (performed here by the French pharmacovigilance Network). As all data recorded in the FPVD are anonymous, informed consent is waived. The FPVD is registered with the French data protection agency (Commission Nationale de l'Informatique et des Libertés, CNIL) (deliberation no. 2014-032, July 10, 2014).

Provenance and peer review Not commissioned; externally peer reviewed.

Data availability statement Data are available upon reasonable request to the corresponding author.

Supplemental material This content has been supplied by the author(s). It has not been vetted by BMJ Publishing Group Limited (BMJ) and may not have been peer-reviewed. Any opinions or recommendations discussed are solely those of the author(s) and are not endorsed by BMJ. BMJ disclaims all liability and responsibility arising from any reliance placed on the content. Where the content includes any translated material, BMJ does not warrant the accuracy and reliability of the translations (including but not limited to local regulations, clinical guidelines, terminology, drug names and drug dosages), and is not responsible for any error and/or omissions arising from translation and adaptation or otherwise.

Open access This is an open access article distributed in accordance with the Creative Commons Attribution Non Commercial (CC BY-NC 4.0) license, which permits others to distribute, remix, adapt, build upon this work non-commercially, 
and license their derivative works on different terms, provided the original work is properly cited, appropriate credit is given, any changes made indicated, and the use is non-commercial. See http://creativecommons.org/licenses/by-nc/4.0/.

\section{ORCID iD}

Mathieu Puyade http://orcid.org/0000-0002-5639-0138

\section{REFERENCES}

1 Hirsch L, Zitvogel L, Eggermont A, et al. PD-Loma: a cancer entity with a shared sensitivity to the PD-1/PD-L1 pathway blockade. $\mathrm{Br} J$ Cancer 2019;120:3-5.

2 Larkin J, Chiarion-Sileni V, Gonzalez R, et al. Five-Year survival with combined nivolumab and ipilimumab in advanced melanoma. $N$ Engl J Med 2019;381:1535-46.

3 Motzer RJ, Escudier B, George S, et al. Nivolumab versus everolimus in patients with advanced renal cell carcinoma: updated results with long-term follow-up of the randomized, open-label, phase 3 CheckMate 025 trial. Cancer 2020;126:4156-67.

4 Horn L, Spigel DR, Vokes EE, et al. Nivolumab versus docetaxel in previously treated patients with advanced non-small-cell lung cancer: two-year outcomes from two randomized, open-label, phase III trials (CheckMate 017 and CheckMate 057). J Clin Oncol 2017;35:3924-33.

5 Sharma P, Retz M, Siefker-Radtke A, et al. Nivolumab in metastatic urothelial carcinoma after platinum therapy (CheckMate 275): a multicentre, single-arm, phase 2 trial. Lancet Oncol 2017;18:312-22.

6 Beköz H, Karadurmus N, Paydas S, et al. Nivolumab for relapsed or refractory Hodgkin lymphoma: real-life experience. Ann Oncol 2017;28:2496-502.

7 Overman MJ, McDermott R, Leach JL, et al. Nivolumab in patients with metastatic DNA mismatch repair-deficient or microsatellite instability-high colorectal cancer (CheckMate 142): an open-label, multicentre, phase 2 study. Lancet Oncol 2017;18:1182-91.

8 Schmid P, Cortes J, Pusztai L, et al. Pembrolizumab for early triplenegative breast cancer. N Engl J Med 2020;382:810-21.

9 Mansfield AS, Każarnowicz A, Karaseva N, et al. Safety and patientreported outcomes of atezolizumab, carboplatin, and etoposide in extensive-stage small-cell lung cancer (IMpower133): a randomized phase I/III trial. Ann Oncol 2020;31:310-7.

10 Postow MA, Sidlow R, Hellmann MD. Immune-Related adverse events associated with immune checkpoint blockade. N Engl J Med 2018;378:158-68.

11 Brahmer JR, Lacchetti C, Schneider BJ, et al. Management of immune-related adverse events in patients treated with immune checkpoint inhibitor therapy: American Society of clinical oncology clinical practice guideline. J Clin Oncol 2018;36:1714-68.

12 Champiat S, Lambotte O, Barreau E, et al. Management of immune checkpoint blockade dysimmune toxicities: a collaborative position paper. Ann Oncol 2016;27:559-74.

13 Haanen JBAG, Carbonnel F, Robert C, et al. Management of toxicities from immunotherapy: ESMO clinical practice guidelines for diagnosis, treatment and follow-up. Ann Oncol 2018;29:iv264-6.

14 Michot JM, Bigenwald C, Champiat S, et al. Immune-related adverse events with immune checkpoint blockade: a comprehensive review. Eur J Cancer 2016;54:139-48.

15 Santini FC, Rizvi H, Plodkowski AJ, et al. Safety and efficacy of Retreating with immunotherapy after immune-related adverse events in patients with NSCLC. Cancer Immunol Res 2018;6:1093-9.

16 Abou Alaiwi S, Xie W, Nassar AH, et al. Safety and efficacy of restarting immune checkpoint inhibitors after clinically significant immune-related adverse events in metastatic renal cell carcinoma. $J$ Immunother Cancer 2020;8:e000144.

17 Dolladille C, Ederhy S, Sassier M, et al. Immune checkpoint inhibitor rechallenge after immune-related adverse events in patients with cancer. JAMA Oncol 2020;6:865-7.

18 Simonaggio A, Michot JM, Voisin AL, et al. Evaluation of Readministration of immune checkpoint inhibitors after immunerelated adverse events in patients with cancer. JAMA Oncol 2019;5:1310.

19 Pollack $\mathrm{MH}$, Betof $\mathrm{A}$, Dearden $\mathrm{H}$, et al. Safety of resuming antiPD-1 in patients with immune-related adverse events (irAEs) during combined anti-CTLA-4 and anti-PD1 in metastatic melanoma. Ann Oncol 2018;29:250-5.

20 Nakajima EC, Lipson EJ, Brahmer JR. Challenge of rechallenge: when to resume immunotherapy following an immune-related adverse event. J Clin Oncol 2019;37:2714-8.

21 Bégaud B, Evreux JC, Jouglard J, et al. [Imputation of the unexpected or toxic effects of drugs. Actualization of the method used in France]. Therapie 1985;40:111-8.

22 Brown EG, Wood L, Wood S. The medical dictionary for regulatory activities (MedDRA). Drug Saf 1999;20:109-17.

23 Abu-Sbeih H, Ali FS, Naqash AR, et al. Resumption of immune checkpoint inhibitor therapy after immune-mediated colitis. J Clin Oncol 2019;37:2738-45.

24 Delyon J, Lourenço N, Vu L-T, et al. Recurrence of immune-mediated colitis upon immune checkpoint inhibitor resumption: does time matter? J Clin Oncol 2019;37:3563-4.

25 Hazell L, Shakir SAW. Under-reporting of adverse drug reactions : a systematic review. Drug Saf 2006;29:385-96.

26 Lopez-Gonzalez E, Herdeiro MT, Figueiras A. Determinants of underreporting of adverse drug reactions: a systematic review. Drug Saf 2009;32:19-31. 